\title{
Photoinduced electron transfer in rhodamine B-containing amorphous titania
}

$$
\text { gels }
$$

Hiromasa Nishikiori,* Katsuya Teshima

Department of Environmental Science and Technology, Faculty of Engineering, Shinshu University, 4-17-1 Wakasato, Nagano 380-8553, Japan

Tsuneo Fujii

Nagano Prefectural Institute of Technology, 813-8 Shimonogo, Ueda, Nagano 386-1211, Japan

Corresponding author: Hiromasa Nishikiori

Tel: +81-26-269-5536

Fax: +81-26-269-5531

E-mail: nishiki@shinshu-u.ac.jp

Department of Environmental Science and Technology, Faculty of Engineering,

Shinshu University, Wakasato, Nagano 380-8553, Japan 


\begin{abstract}
Amorphous titania gel films containing the dye rhodamine $\mathrm{B}(\mathrm{RhB})$ were prepared by a sol-gel method without heating. The $\mathrm{RhB}$ existed in the films as the $\mathrm{RhB}$ cation, which was transformed into the rhodamine 110 (Rh110) cation; its xanthene skeleton then decomposed during irradiation with visible light. This process was induced by electron transfer from the RhB cation to the titania gel matrix, because formation of the $\mathrm{RhB}$ radical on loss of an electron caused sequential dissociation of the $N$-ethyl groups. During irradiation with visible light a photocurrent was observed in electrodes coated with the RhB-containing titania gel and immersed in an $\mathrm{I}_{2}-\mathrm{LiI}$ electrolyte. This photocurrent resulted from electron injection from the LUMO level of the dye into conduction band-like states of the titania gel. Because of its effective electron supply the electrolyte inhibited dissociation of the $N$-ethyl groups of RhB.
\end{abstract}

Keywords: rhodamine B, photoinduced electron transfer, photocurrent, amorphous gel; titania 


\section{Introduction}

Xanthene dyes are promising photofunctional materials for harvesting and emitting light energy, because of their efficient absorption and fluorescence of visible light [1]. For practical use of such dyes, it is convenient if the dye molecules are encapsulated in inorganic matrices which are resistant to heat and light irradiation [2-6]. For such encapsulation both organic and inorganic substances are used, because of the advantages of each. In many cases, an amorphous or crystalline thin film is required when applying dyes to such materials. It is useful to prepare dip-coated thin films containing the organic dyes by use of the sol-gel method [7-11], which easily enables preparation of materials consisting of amorphous or crystalline states. Thus, an organic dye with a high photofunction can be encapsulated in the small pores of inorganic matrices $[2-5,12,13]$. Compared with conventional materials on which dyes are adsorbed, the dye molecules in such materials absorb and fluoresce more efficiently, because they are finely dispersed in the amorphous or nanosized crystalline matrices [14,15]. It is expected that such films will have more applications and their performance will be more stable in a variety of applications.

Both amorphous and crystalline titania can be n-type semiconductors [14-19]. Aanatase-type crystals frequently have high photoinduced activity. Thin sol-gel films prepared from titanium alkoxide are heated and form an anatase-type crystal for use as functional materials in photocatalysts and solar cells [16-18,20,21]. Because films containing dyes can be used as photofunctional materials, it is important to study changes in the molecular forms of the dyes in thin sol-gel films during light irradiation. It has been reported that the thermo-stability and photo-stability of organic dyes are improved by encapsulating the dyes in sol-gel matrices [2-6]. 
In a previous study it was shown that rhodamine $\mathrm{B}(\mathrm{RhB})$ molecules interact strongly with -TiOH groups in thin sol-gel silica-titania films [22]. Negishi et al. [23] reported that RhB molecules encapsulated in a bulk gel, by use of the sol-gel reaction of silicon and titanium alkoxides, interacted strongly with the $-\mathrm{TiOH}$ group. Watanabe et al. [24] reported that $\mathrm{RhB}$ in a CdS-suspended solution is transformed into $N$-deethylated rhodamine 110 (Rh110) during visible light irradiation (Scheme 1). This was confirmed photoelectrochemically [25]. $N$-Deethylation of $\mathrm{RhB}$ has also been observed on crystalline titania particles during irradiation with visible light [26-30]. However, the reaction of rhodamines encapsulated in amorphous titania gels has not been reported.

In this study, thin amorphous gel films of silica, titania, and silica-titania containing RhB or Rh110 were prepared by use of the sol-gel reaction of tetraethyl orthosilicate (TEOS) and/or titanium tetraisopropoxide (TTIP). The matrix properties of the titania gel were compared with those of the other gels. The effect of irradiation with light on the molecular forms of the dyes, which interact with the matrix and transfer electrons to it, was investigated by steady state and time-resolved fluorescence analysis and use of photoelectric measurements.

\section{Experimental}

\section{Materials}

RhB, ethanol, TEOS, TTIP, hydrochloric acid, ethylene glycol, iodine, and lithium iodide (Wako, S or reagent grade), and Rh110 (Indeco, laser grade) were used without further purification. Water was deionized and distilled. Glass plates for use as the substrate (Matsunami S-1126) were washed with neutral detergents, soaked in hydrochloric acid (0.1 mol

$\mathrm{dm}^{-3}$ ) for $1 \mathrm{~h}$, washed with water, and finally dried at room temperature. Glass plates coated 
with the ITO transparent electrode (AGC Fabritec) were soaked in hydrochloric acid (1.0 mol $\mathrm{dm}^{-3}$ ) for $1 \mathrm{~h}$ and then rinsed with water. The electrolyte used for electrical measurements was a solution of iodine $\left(5.0 \times 10^{-2} \mathrm{~mol} \mathrm{dm}^{-3}\right)$ and lithium iodide $\left(0.50 \mathrm{~mol} \mathrm{dm}^{-3}\right)$ in ethylene glycol.

\section{Sample preparation}

1) The sol-gel starting solution of TEOS (System Ti0) contained the RhB or Rh110 ethanol solution $\left(1.0 \times 10^{-2} \mathrm{~mol} \mathrm{dm}{ }^{-3}, 21.0 \mathrm{~cm}^{3}\right)$, TEOS $\left(20.0 \mathrm{~cm}^{3}=8.95 \times 10^{-2} \mathrm{~mol}\right)$, and hydrochloric acid $\left(1.0 \times 10^{-5} \mathrm{~mol} \mathrm{dm}{ }^{-3}, 6.5 \mathrm{~cm}^{3}\right)$ as catalyst. This solution was stirred during addition then stirred thoroughly for an additional $30 \mathrm{~min}$. 2) The sol-gel starting solution of TTIP (System Ti100) contained the RhB or Rh110 ethanol solution $\left(1.0 \times 10^{-2} \mathrm{~mol} \mathrm{dm}{ }^{-3}, 21.0 \mathrm{~cm}^{3}\right)$, TTIP $(20.0$ $\left.\mathrm{cm}^{3}=8.95 \times 10^{-2} \mathrm{~mol}\right)$, and hydrochloric acid $\left(1.0 \times 10^{-3} \mathrm{~mol} \mathrm{dm}^{-3}, 0.040 \mathrm{~cm}^{3}\right)$ as catalyst.

The sol-gel starting solutions of the TEOS-TTIP binary (Systems Ti25, Ti50, and Ti75) contained the $\mathrm{RhB}$ ethanol solution $\left(1.0 \times 10^{-2} \mathrm{~mol} \mathrm{dm} \mathrm{dm}^{-3}, 21.0 \mathrm{~cm}^{3}\right)$, metal alkoxides $\left(\right.$ TEOS:TPOT $=75: 25,50: 50$, or 25:75, TEOS + TPOT $\left.=8.95 \times 10^{-2} \mathrm{~mol}\right)$, and hydrochloric acid $\left(1.0 \times 10^{-3} \mathrm{~mol} \mathrm{dm}^{-3}, 0.040 \mathrm{~cm}^{3}\right)$ as catalyst. The materials, except for TTIP, were stirred during addition and thoroughly for an additional $10 \mathrm{~min}$, and were then mixed with TTIP under a dry $\mathrm{N}_{2}$ atmosphere and stirred for a further $10 \mathrm{~min}$.

These mixed solutions were poured into individual polypropylene vials $\left(50 \mathrm{~cm}^{3}\right)$. The vials were closed with a cover containing a hole and kept in a thermostated chamber at $50{ }^{\circ} \mathrm{C}$. The glass plates and the glass plates with ITO were vertically withdrawn from the sol-gel solution in the vial at a speed of $10 \mathrm{~mm} \mathrm{~min}^{-1}$ at $25{ }^{\circ} \mathrm{C}$ and relative humidity $60 \%$. The dip-coated thin films were made at half the gelation time of the individual sol-gel starting solutions. The prepared films were aged at $35{ }^{\circ} \mathrm{C}$ and relative humidity $20 \%$ for 5 days. The relative humidity was controlled by use of a Sanplatec STC-1 constant temperature and humidity 
chamber.

\section{Spectral measurements}

UV-visible absorption and fluorescence spectra were acquired by use of a Shimadzu UV-2500 spectrophotometer and a Shimadzu RF-5300 fluorescence spectrophotometer, respectively. Irradiation was performed with a $150 \mathrm{~W}$ Xe short arc lamp (Hamamatsu Photonics L2175) with a cut-off filter for visible light irradiation $(\lambda>430 \mathrm{~nm})$. Absorption of thin films containing RhB and Rh110 was measured as a function of irradiation time.

Flakes of the dye-containing film samples were pressed in $\mathrm{KBr}$ pellets and their IR spectra were obtained by use of a Shimadzu FTIR-8300 FTIR spectrophotometer.

A Ti:Sapphire femtosecond pulse laser and streak scope spectroscopic system were used for time resolved fluorescence measurements, to obtain information about fluorescence quenching because of electron injection from the dye to the titania [15,31]. The laser system (Clark MXR CPA 2001) generates laser pulses of 150 fs duration (FWHM) with an energy of $750 \mu \mathrm{J}$ at 750 $\mathrm{nm}$ at a repetition rate of $1 \mathrm{kHz}$. The excitation source was tuned to a wavelength of $540 \mathrm{~nm}$ and FWHM of 250 fs by use of an infrared optical parametric amplifier (Clark-MXR, IR-OPA) with a fourth-harmonic generator. Fluorescence signal was monitored by use of a streak scope system (Hamamatsu Photonics C4780). Fluorescence decay curves were obtained by integrating the fluorescence signals in the 570-620 $\mathrm{nm}$ region.

The iodine-based electrolyte was left to soak into the space between the electrode sample and the counter $\mathrm{Pt}$ electrode. Monochromatic light obtained from the fluorescence spectrophotometer with a $150 \mathrm{~W}$ Xe short arc lamp (Ushio UXL-155) was used to irradiate the electrodes for the spectroscopic measurements. Under light irradiation, the short circuit currents of the electrodes were measured by use of an electrometer (Keithley model 617). To estimate the incident photon to current conversion efficiency (IPCE) the intensity at each 
wavelength of the light source was obtained by use of a power meter (Molectron PM500A). The light intensity was confirmed to correlate with results from potassium ferrioxalate actinometry.

\section{Results and discussion}

\section{Changes in molecular forms of rhodamine $B$ in gel films during visible light irradiation}

Figure 1 shows the UV-visible absorption spectra of RhB in thin films containing silica and titania gels in different $\mathrm{Si} / \mathrm{Ti}$ molar ratios. For all the gel films, the absorption peak was observed at approximately $560 \mathrm{~nm}$; this which was assigned to the $\mathrm{RhB}$ cation monomer [12]. The peak wavelength was slightly dependent on the composition of silica and titania. A small amount of the dimer was also observed in these films. Absorbance by the thin films depended on their thickness, which was mainly determined by the viscosity of the sols. Because of its thickness, the absorbance of the silica gel film was much higher those of the others (Scheme 1).

Figure 2 shows the fluorescence spectra of $\mathrm{RhB}$ in thin films containing the silica and titania gels, and their peak intensities. For all the films, the peak was observed at approximately $585 \mathrm{~nm}$; this was assigned to the RhB cation monomer [12]. The fluorescence intensity values were divided by the maximum absorbance for each film to enable comparison of their fluorescence quantum yields, i.e., the intensity indicated the relative fluorescence quantum yields. The relative fluorescence quantum yields decreased with increasing the titanium content. The $\mathrm{Si}-\mathrm{O}$ and $\mathrm{Ti}-\mathrm{O}$ networks are homogeneously mixed in silica-titania gels prepared by the sol-gel method with up to ca $40 \%$ titania $[32,33]$. $\mathrm{TiO}_{4}$ tetrahedra are homogeneously dispersed in the silica-titania gels with low titania content. $\mathrm{TiO}_{6}$ octahedra are formed, and aggregate in gels with high titania content. It is possible that parts of the gel containing the $\mathrm{Ti}-\mathrm{O}$ networks caused the fluorescence quenching of RhB by electron transfer from the excited $\mathrm{RhB}$ cation to the titania gel. 
Titania gel may have a Ti3d orbital potentially suitable for oxidation of the RhB, similar to the conduction band of anatase titania $[14,15,19]$.

Figure 3 shows the effect of visible light irradiation time on the spectrum of RhB in the silica and titania gels, In the silica gel film, the absorption peak at approximately $559 \mathrm{~nm}$ before light irradiation was shifted to higher energy, to $543 \mathrm{~nm}$ and its intensity gradually decreased with increasing irradiation time. It is suggested that the $\mathrm{RhB}$ cation was transformed to the lactone form via the zwitterion and then decomposed as a result of oxidative photolysis [34,35]. In the titania gel film, the spectrum was somewhat broader than that in the silica gel film because the $\mathrm{RhB}$ cation interacted with the $-\mathrm{TiOH}$ group. The peak shifted substantially, from approximately $560 \mathrm{~nm}$ to approximately $510 \mathrm{~nm}$ on irradiation with light for $1 \mathrm{~min}$, indicating dissociation of the $N$-ethyl groups and transformation into the Rh110 cation which interacted with the -TiOH group [24]. In water the Rh110 cation has an absorption peak at $498 \mathrm{~nm}$. The intensity of the $510 \mathrm{~nm}$ band gradually decreased with further irradiation. The Rh110 cation was transformed to the lactone form and then decomposed.

The change of the spectrum of Rh110 in the thin films was also examined to confirm the above reaction. Figure 4 show the change of the spectrum of Rh110 in the silica and titania films during visible light irradiation. In the silica gel, the absorption peak of the Rh110 cation found at $495 \mathrm{~nm}$ gradually decreased without any spectral shift during irradiation with light. In the titania gel, the R110 cation interacting with the $-\mathrm{TiOH}$ group had an absorption peak at approximately $510 \mathrm{~nm}$ before light irradiation. The absorbance gradually decreased without any spectral shift on irradiation with light. All the changes of the Rh110 spectrum were indicative of transformation from the cationic form to the lactone via the zwitterion and subsequent decomposition. Dissociation of the $N$-ethyl groups promoted decomposition of the xanthene chromophore skeleton; decay of the absorption of RhB was faster than that of Rh110. 
Figure 5 shows the IR spectra of $\mathrm{RhB}$ and R110 in the titania gel films before and after irradiation with visible light for $5 \mathrm{~min}$. The IR spectrum of $\mathrm{RhB}$ observed before light irradiation contained bands at approximately 3,400 and $2,980-2,870 \mathrm{~cm}^{-1}$, which can be assigned to the $\mathrm{OH}$ stretching vibration of the $-\mathrm{TiOH}$ groups on the titania surface and the $\mathrm{CH}$ stretching vibration of the $\mathrm{N}$-ethyl groups of $\mathrm{RhB}$, respectively. The $\mathrm{NH}$ stretching vibration of the amino groups of Rh110 was observed at approximately 3,300 and $3,200 \mathrm{~cm}^{-1}$ before light irradiation. In the RhB-containing film, the relative intensity of the $\mathrm{CH}$ bands decreased and that of the amino groups increased during irradiation, indicating that some of the $\mathrm{RhB}$ molecules were $N$-deethylated and transformed to Rh110. On the other hand, only a slight peak shift was observed for the spectrum of the Rh110-containing film during irradiation.

Figure 6 shows the absorbance decay of RhB vs. visible light irradiation time in thin films containing silica and titania gels with different $\mathrm{Si} / \mathrm{Ti}$ ratios. The absorbance of the individual films observed at $550 \mathrm{~nm}$ was normalized to the absorbance before the light irradiation. The rate of decay was faster with increasing titanium content, indicating the $\mathrm{RhB}$ cation was transformed faster into the Rh110 cation and the lactone form. Decay was especially significantly accelerated in systems containing with more than $25 \%$ titania. Although the $\mathrm{TiO}_{4}$ tetrahedra are homogeneously dispersed in the systems with low titania content (ca. $<40 \%$ ), a larger number of the $\mathrm{TiO}_{6}$ octahedral aggregates are formed in systems with higher titania content [32,33]. It is suggested that the efficiency of electron transfer from the RhB cation to the matrix increased with increasing amounts of Ti-O networks because the dye molecules interact strongly with the - $\mathrm{TiOH}$ groups and the $\mathrm{TiO}_{6}$ octahedral networks had a semiconductor-like conduction band $[14,15,19]$. In addition, the charge separation lifetime should be an important factor determining the rate of photoreaction, which depends on the density of the conduction band, density, i.e., the 
concentrations of electrons and holes in the steady state during irradiation.

Watanabe et al. showed that RhB in a CdS-suspended solution was transformed into R110 during irradiation with visible light because formation of the $\mathrm{RhB}$ radical upon loss of electron caused the sequential dissociation of the $N$-ethyl groups accompanying the formation of acetaldehyde [24]. Chen et al. [27] showed the highly selective stepwise deethylation process of RhB before destruction of the chromophore structure during visible light irradiation on $\mathrm{TiO}_{2}-\mathrm{SiO}_{2}$ composite, which adsorbed the $\mathrm{RhB}$ molecules through the positively charged diethylamino group. Therefore, dissociation of the $N$-ethyl groups from $\mathrm{RhB}$ occurred readily when its diethylamino groups were adsorbed on the negatively charged titania surface, which was formed by introduction of negative species [26-29]. Deethylation of $\mathrm{RhB}$ requires a strong interaction between the diethylamino groups and the titania surface. In this titania gel, this reaction was also induced by electron transfer from the $\mathrm{RhB}$ cation to the matrix during irradiation with visible light. Dissociation of the ethyl groups from the resulting RhB radical was caused by the reaction with the active oxygen species on the titania gel surface, for example the superoxide radical, hydrogen peroxide, and/or hydroxyl radical, which were formed from the oxygen molecule after trapping of the electrons in the conduction band-like states. In these present systems, the RhB cation existed in the small pores of the gels and was surrounded by the matrix networks. Therefore, the diethylamino groups easily interacted with the active parts of the matrix, irrespective of the matrix charge of the silica and titania. It is also suggested that the longer lifetime of the charge separation induced the dissociation of a greater number of $N$-ethyl groups. In these titania gels, the $\mathrm{RhB}$ cation was tightly encapsulated in the titania matrix and easily induced injection of the electron.

\section{Electron transfer from rhodamine $B$ to the gel matrices by visible light irradiation}


The fluorescence decay curves of $\mathrm{RhB}$ in the gel films were obtained by the time-resolved fluorescence measurement; the results are shown in Fig. 7. The intensity values were divided by the maximum absorbance of each film to enable comparison of their fluorescence quantum yields. The curves were fitted to the double exponential decay. The fluorescence intensities just after excitation, i.e., the maximum intensities, and the fitting date are listed in Table 1. The decay curves consist of two components with lifetimes of $0.16-0.22$ and 3.7-4.3 ns. These values correspond to those reported for titania systems with absorbed dye [36]. The longer lifetime component is assigned to the original $\mathrm{RhB}$ cation. The fluorescence lifetime of the cation in the silica gel was reported to be ca. 3.5-4.0 ns $[23,37,38]$. The shorter lifetime component can be assigned to the fluorescence of the dye interacting with the matrix. The literature states that $\mathrm{RhB}$ interacting weakly with the silica matrix had a fluorescence lifetime of ca $0.2-0.3 \mathrm{~ns}[23,36]$. The $\mathrm{RhB}$ cation interacts strongly with the $-\mathrm{TiOH}$ group in the gel films. The lifetimes were reduced by increasing the titanium content, because of the increase in the amount of $-\mathrm{TiOH}$. Weaker maximum fluorescence intensity was observed for the electrode containing more titanium. The yield of electron injection from the dye to the titania can have an inverse relationship with the maximum intensity of fluorescence because electron injection is reported to occur within 100 ps $[31,39,40]$. Therefore, the relative electron injection yield in the dye-containing gel increased with increasing titanium content not only because of the strong interaction between the $\mathrm{RhB}$ cation and the $-\mathrm{TiOH}$ group but also because the Ti3d-orbital potential was suitable for oxidation of the excited RhB.

Figures 8 and 9 show the IPCE spectra of the RhB-containing and Rh110-containing titania gel electrodes and their absorption spectra observed before and after the photoelectric 
measurement. The absorption spectrum of $\mathrm{RhB}$ was red-shifted by irradiation with light for the measurement, by an amount similar to that shown in Fig. $3 b$, because of transformation into Rh110. The shape of the IPCE spectrum of RhB was broad over the region of the absorption spectra observed before and after the measurement. On the other hand, the absorption spectrum of Rh110 was slightly shifted by the measurement and the IPCE spectrum corresponded to the shape of the absorption spectra. The photocurrent was indicative of electron injection from the LUMO of the dyes to the conduction band-like states of the titania gel. We have previously confirmed that amorphous titania gels prepared by the sol-gel method without heating have such electron states $[19,41]$. Although the photocurrent quantum efficiency was very low in these electrodes, it was increased substantially by steam treatment, because of crystallization and densification of the amorphous titania compared with that of the untreated electrodes and the conventional dye-adsorbed electrodes [14,15].

The LUMO levels of such xanthene dyes are reported to be not significantly different, i.e., -0.58 to -0.55 versus the normal hydrogen electrode (NHE) [42,43]. The LUMO levels of the xanthene dyes can be more negative than the level of the titania conduction band edge (ca -0.5 vs. NHE) [44]. The titania gel has a Ti3d-orbital potential close to the conduction band edge of anatase titania $[14,15,19]$. Therefore, the electrons in the LUMO of the dyes can be injected to the conduction band-like states of the titania gel.

\section{(Figures 8 and 9)}

Figure 10 shows the change in the relative absorbance of $\mathrm{RhB}$ in the titania gel films induced by visible light irradiation; this was observed in the presence and absence of electrolyte. The absorbance decay was mainly caused by the transformation into Rh110, which was very fast process, as shown in Fig 3b. The decay rate observed in the electrolyte was clearly slower than that observed without the electrolyte. Rapid reduction of the oxidized dye is required for the 
continuous electron cycle in the dye-sensitized solar cells. The electrolyte inhibited dissociation of the $N$-ethyl groups of RhB because of its effective electron supply.

(Figure 10)

\section{Conclusions}

The silica, titania, and silica-titania gel thin films including RhB or Rh110 were prepared using the sol-gel reaction of TEOS and/or TTIP. The effect of irradiation with light on changes of the molecular forms of the dyes, which interact with the matrix and induce electron transfer to the matrix, was investigated by steady state and time-resolved fluorescence analysis and photoelectric measurements. $\mathrm{RhB}$ existed in the titania-containing films as the $\mathrm{RhB}$ cation, which was transformed into the Rh110 cation; its xanthene skeleton then decomposed during irradiation with visible light. The RhB cation in the silica gel films was decomposed without transformation into Rh110. The Rh110 also existed in all the films as the cation and its xanthene skeleton was decomposed during irradiation with visible light. This process was induced by electron transfer from the $\mathrm{RhB}$ cation to the titania gel matrix, because formation of the $\mathrm{RhB}$ radical upon loss of an electron caused sequential dissociation of the $N$-ethyl groups. During irradiation with visible light a photocurrent was observed in ITO electrodes coated with the RhB or Rh110-containing titania gel immersed in $\mathrm{I}_{2}-\mathrm{LiI}$ electrolyte. This photocurrent resulted from electron injection from the LUMO level of the dye into conduction band-like states of the titania gel. The electrolyte inhibited dissociation of the $N$-ethyl groups of RhB because of its effective electron supply.

Acknowledgements This work was supported by JSPS KAKENHI grant number 24550153. 


\section{References}

1. M. Maeda, Laser Dyes: Properties of Organic Compounds for Dye Lasers (Academic Press, Tokyo, 1984)

2. D. Avnir, D. Levy, R. Reisfeld, J. Phys. Chem. 88, 5956 (1984)

3. R. Reisfeld, R. Zusman, Y. Cohen, M. Eyal, Chem. Phys. Lett. 147, 142 (1988)

4. T. Fujii, A. Ishii, M. Anpo, J. Photochem. Photobiol. A. 54, 231 (1990)

5. U. Narang, F.V. Bright, P.N. Prasad, Appl. Spectrosc. 47, 229 (1993)

6. P.H. Chang, H.C. Tsai, Y.R. Chen, J.Y. Chen, G.H. Hsiue, Langmuir 24, 11921 (2008)

7. H. Dislich, Angew. Chem. Int. Ed. Engl. 10, 363 (1971).

8. H. Dislich, J. Non-Cryst. Solids 57, 371 (1983)

9. C.J. Brinker, G.W. Scherer, Sol-Gel Science: The Physics and Chemistry of Sol-Gel Processing (Academic Press, San Diego, 1990)

10. C.J. Brinker, G.C. Frye, A.J. Hurd, C.S. Ashley, Thin Solid Films 201, 97 (1991)

11. C.J. Brinker, A.J. Hurd, G.C. Frye, P.R. Schunk, C.S. Ashley, J. Ceram. Soc. Jpn. 99, 862 (1991)

12. H. Nishikiori, T. Fujii, J. Phys. Chem. B 101, 3680 (1997)

13. H. Nishikiori, N. Tanaka, Y. Minami, A. Katsuki, T. Fujii, J. Photochem. Photobiol. A. 212, $62(2010)$

14. H. Nishikiori, Y. Uesugi, S. Takami, R.A. Setiawan, T. Fujii, W. Qian, M.A. El-Sayed, J. Phys. Chem. C 115, 2880 (2011)

15. H. Nishikiori, Y. Uesugi, R.A. Setiawan, T. Fujii, W. Qian, M.A. El-Sayed, J. Phys. Chem. C 116, 4848 (2012) 
16. B. O’Regan, M. Grätzel, Nature 353, 737 (1991)

17. M.K. Nazeeruddin, A. Kay, I. Rodicio, R. Hamphry-Baker, E. Müeller, P. Liska, N. Vlachopoulos, M. Grätzel, J. Am. Chem. Soc. 115, 6382 (1993)

18. M. Grätzel, J. Photochem. Photobiol. C 4, 145 (2003)

19. H. Nishikiori, R.A. Setiawan, K. Miyamoto, G. Sukmono, Y. Uesugi, K. Teshima, T. Fujii, RSC Adv. 2, 4258 (2012)

20. A. Fujishima, T.N. Rao, D.A. Tryk, J. Photochem. Photobiol. C. 1, 1 (2000)

21. D. Chatterjee, S. Dasgupta, J. Photochem. Photobiol. C. 6, 186 (2005)

22. H. Nishikiori, N. Tanaka, T. Fujii, J. Sol-Gel Sci. Technol. 20, 95 (2001)

23. N. Negishi, M. Fujino, H. Yamashita, M.A. Fox, M. Anpo, Langmuir, 10, 1772 (1994)

24. T. Watanabe, T. Takizawa, K. Honda, J. Phys. Chem. 81, 1845 (1977)

25. J.M. Austin, I.R. Harrison, T.I. Quickenden, J. Phys. Chem. 90, 1839 (1986)

26. P. Qu, J. Zhao, T. Shen, H. Hidaka, J. Mol. Catal. A 129, 257 (1998)

27. F. Chen, J. Zhao, H. Hidaka, Int. J. Photoenergy 5, 209 (2003)

28. Q. Wang, C. Chen, D. Zhao, W. Ma, J. Zhao, Langmuir 24, 7338 (2008)

29. J. Zhuang, W. Dai, Q. Tian, Z. Li, L. Xie, J. Wang, P. Liu, Langmuir 26, 9686 (2010)

30. G. Zi, Y. Wang, K. Zheng, H. Zhao, F. Wang, W. Zhang, Z. Yan, J. He, J. Wang, Res. Chem. Intermed. 38, $2383(2012)$

31. L. Du, A. Furube, K. Hara, R. Katoh, M. Tachiya, J. Phys.Chem. C 114, 8135 (2010)

32. R. Anderson, G. Mountjoy, M. E. Smith, R. J. Newport, J. Non-Cryst. Solids 232-234, 72 (1998)

33. G. Mountjoy, M.A. Holland, P. Gunawidjaja, G.W. Wallidge, D.M. Pickup, R.J. Newport, M.E. Smith, J. Sol-Gel Sci. Technol. 26, 161 (2003) 
34. R.W. Ramette, E.B. Sandell, J. Am. Chem. Soc. 78, 4872 (1956)

35. I.L. Arbeloa, P.R. Ojeda, Chem. Phys. Lett. 79, 347 (1981)

36. K. Kemnitz, T. Murao, I. Yamazaki, N. Nakashima, K. Yoshihara, Chem. Phys. Lett. 101, $337(1983)$

37. F. del Monte, D. Levy, J. Phys. Chem. B 102, 8036 (1998)

38. M.A. Khader, Optics Laser Technol. 40, 445 (2008)

39. J.B. Asbury, N.A. Anderson, E. Hao, X. Ai, T. Lian, J. Phys. Chem. B 107, 7376 (2003)

40. G. Benkö, J. Kallioinen, J.E.I. Korppi-Tommola, A.P. Yartsev, V. Sundström, A.P. Yartsev, J. Am. Chem. Soc. 124, 489 (2002)

41. H. Nishikiori, N. Tanaka, T. Kitsui, T. Fujii, J. Photochem. Photobiol. A 179, 125 (2006)

42. M.S. Chan, J.R. Bolton, Sol. Energy 24, 561 (1980).

43. J. Yu, T. Zhou, J. Electroanal. Chem. 504, 89 (2001)

44. A. Hagfeldt, M. Grätzel, Chem. Rev. 95, 49 (1995) 


\section{Figure captions}

Fig. $1 \mathrm{UV}-$ visible absorption spectra of $\mathrm{RhB}$ in thin films containing silica and titania gels

Scheme 1 Molecular forms of RhB and Rh110

Fig. 2 Fluorescence spectra of RhB in thin films containing the silica and titania gels. (a), and their peak intensities (b). The excitation wavelength was $540 \mathrm{~nm}$

Fig. 3 Change in the UV-visible absorption spectra of RhB in the a silica and $\mathbf{b}$ titania gel films during visible light irradiation

Fig. 4 Change in UV-visible absorption spectra of Rh110 in the a silica and b titania gel films during visible light irradiation

Fig. 5 FTIR spectra of $(1,2) \operatorname{RhB}$ and $(3,4) \operatorname{Rh} 110$ in the titania gel films $(1,3)$ before and $(2,4)$ after irradiation with visible light for $5 \mathrm{~min}$

Fig. 6 Change in the relative absorbance of $\mathrm{RhB}$ in thin films containing silica and titania gels

during visible light irradiation. The absorbance of each film observed at $550 \mathrm{~nm}$ was normalized to the absorbance before irradiation

Fig. 7 Fluorescence decay curves for $\mathrm{RhB}$ in the thin films containing silica and titania gels

Fig. 8 IPCE spectrum of the RhB-containing titania gel electrode and its absorption spectra observed before and after photoelectric measurement

Fig. 9 IPCE spectrum of the Rh110-containing titania gel electrode and its absorption spectra observed before and after photoelectric measurement

Fig. 10 Changes in the relative absorbance of $\mathrm{RhB}$ in titania gel films during visible light irradiation, which were observed in the presence and absence of electrolyte. The absorbance of each film observed at $550 \mathrm{~nm}$ was normalized to the absorbance before the irradiation 
Scheme 1<smiles>CC=[N+]=c1ccc2c(-c3ccccc3C(=O)O)c3ccc(N(CC)CC)cc3oc-2c1</smiles>

$\mathrm{RhB}$<smiles>Nc1ccc2c(-c3ccccc3C(=O)O)c3ccc(=[NH2+])cc-3oc2c1</smiles>

Rh110 
Figure 1

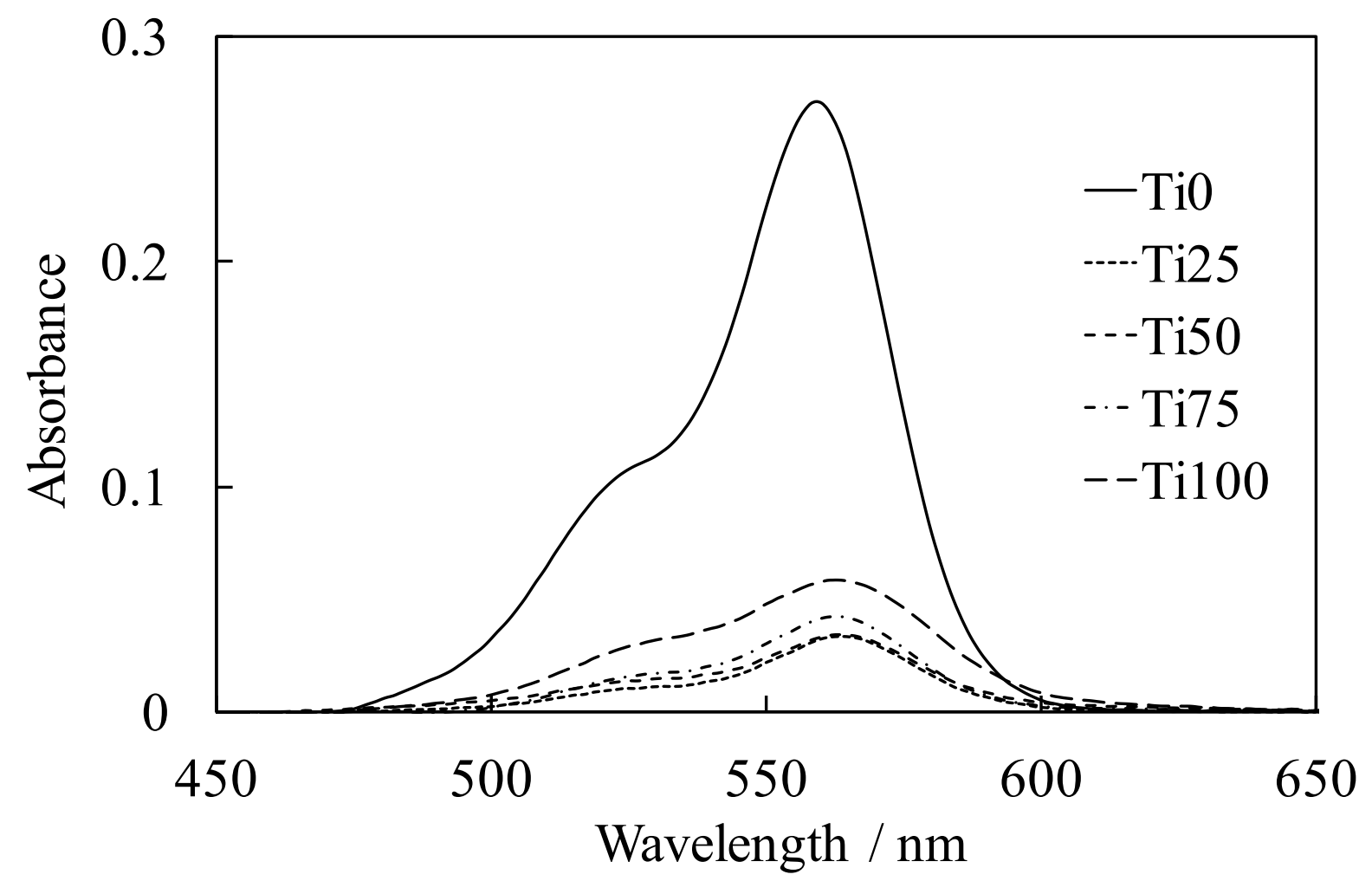


Figure 2
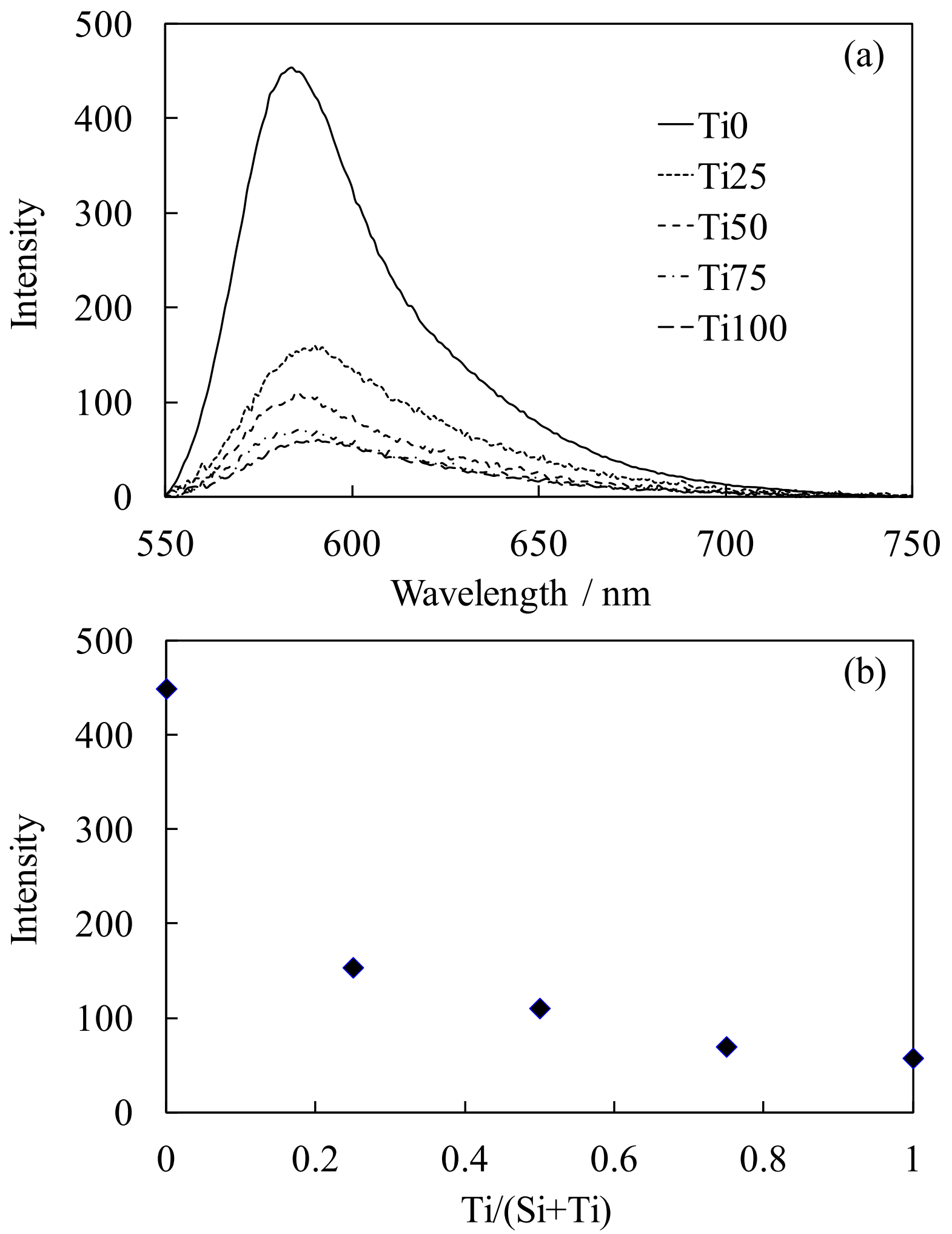
Figure 3

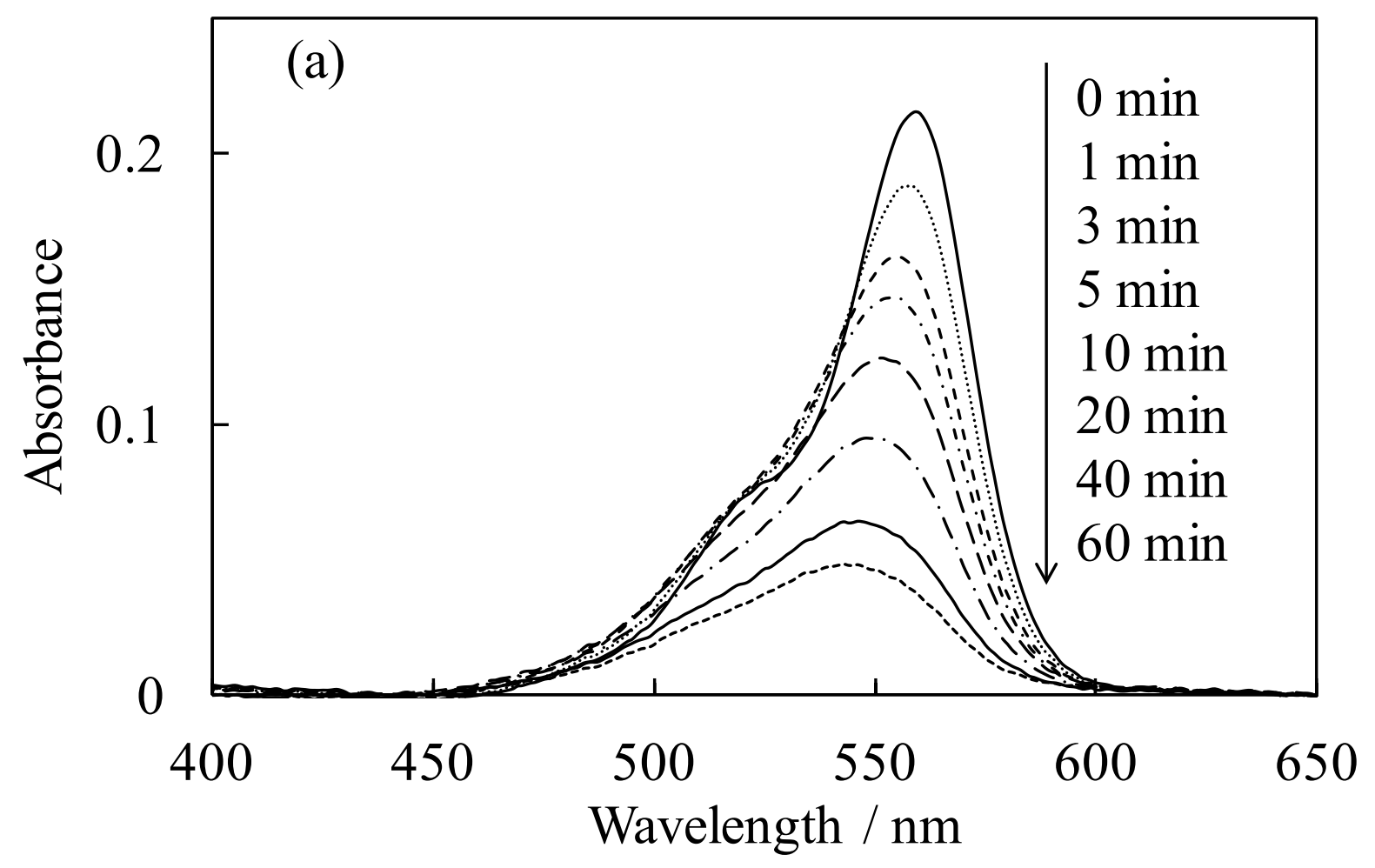




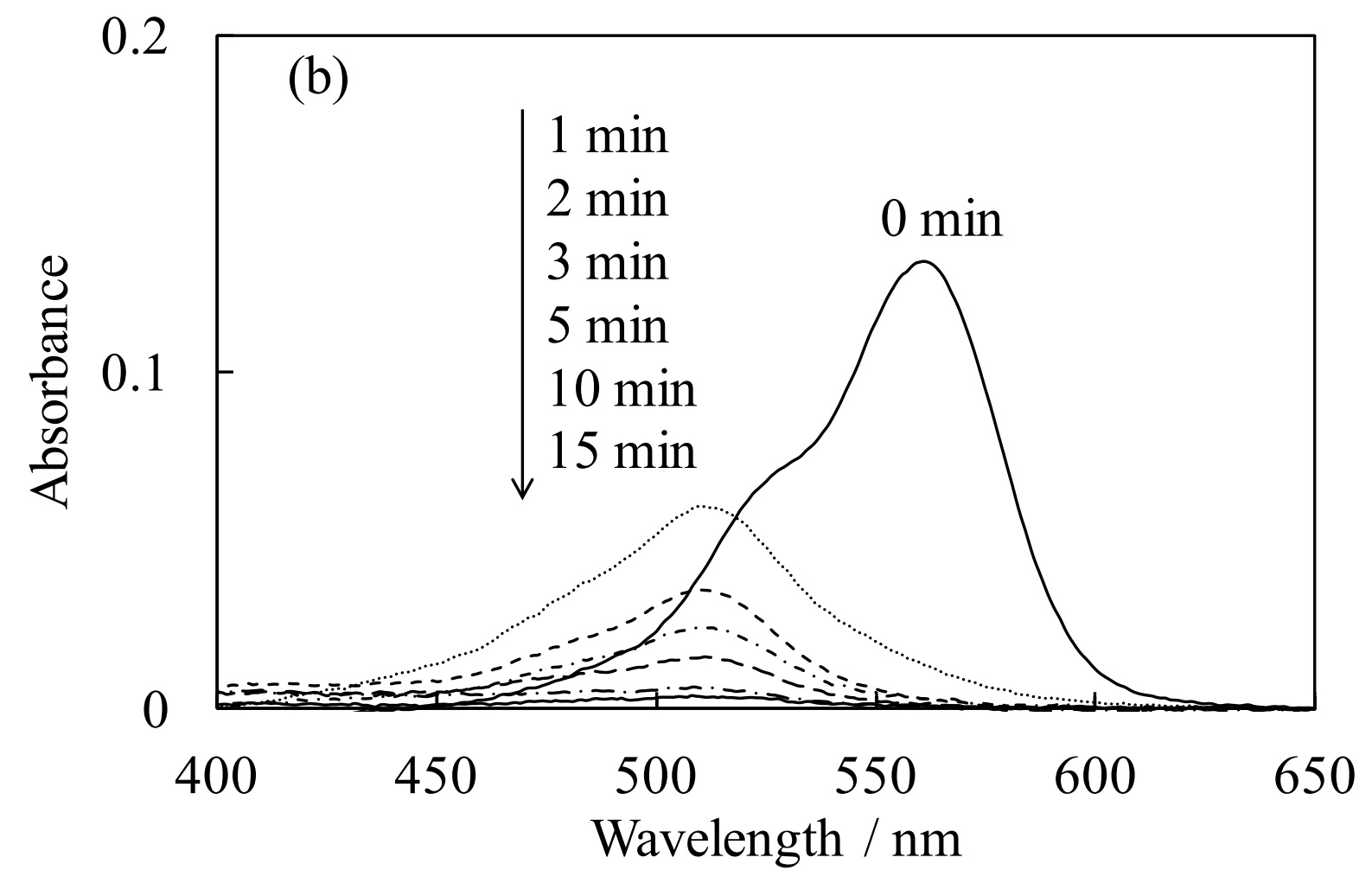


Figure 4

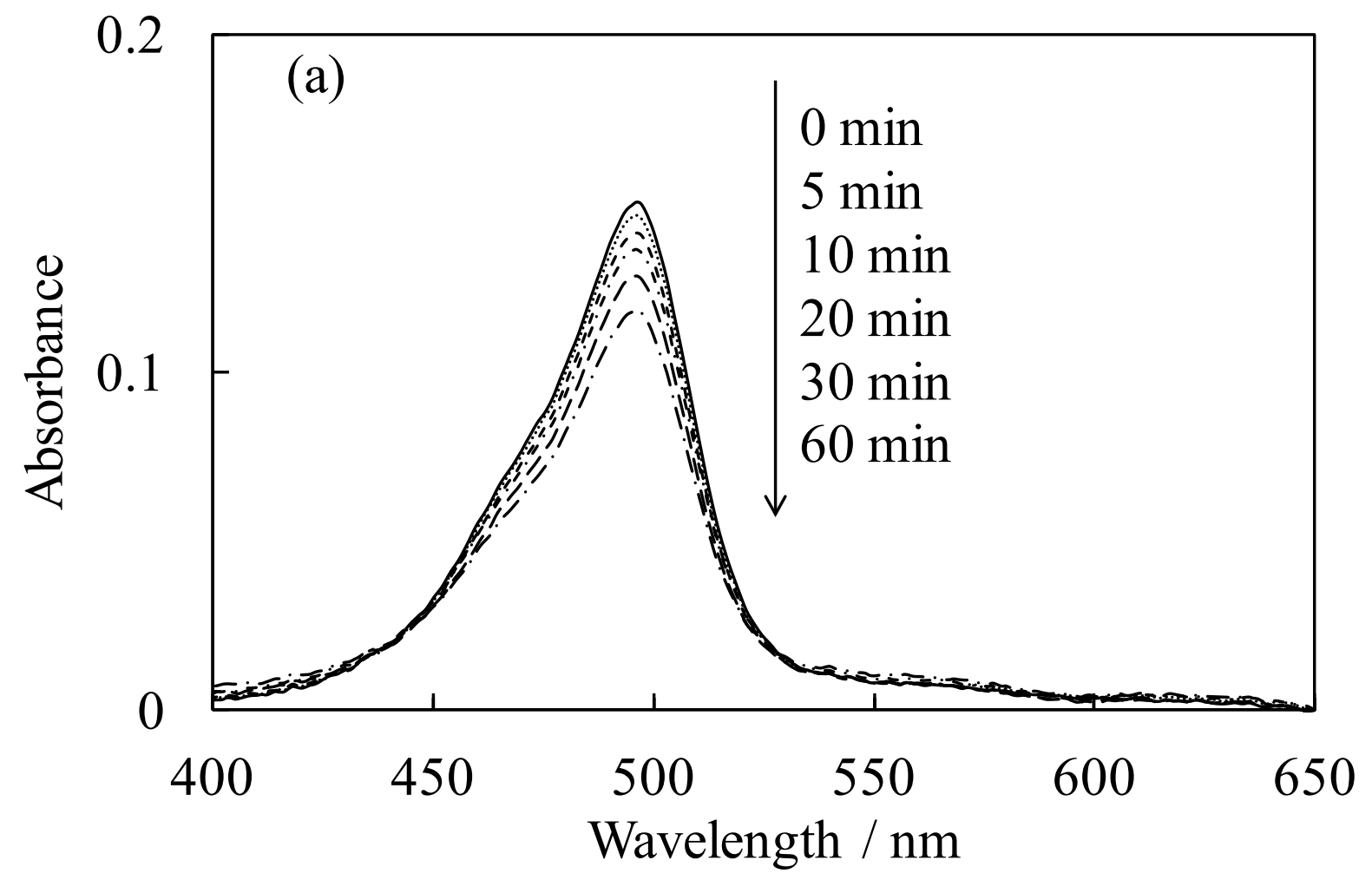




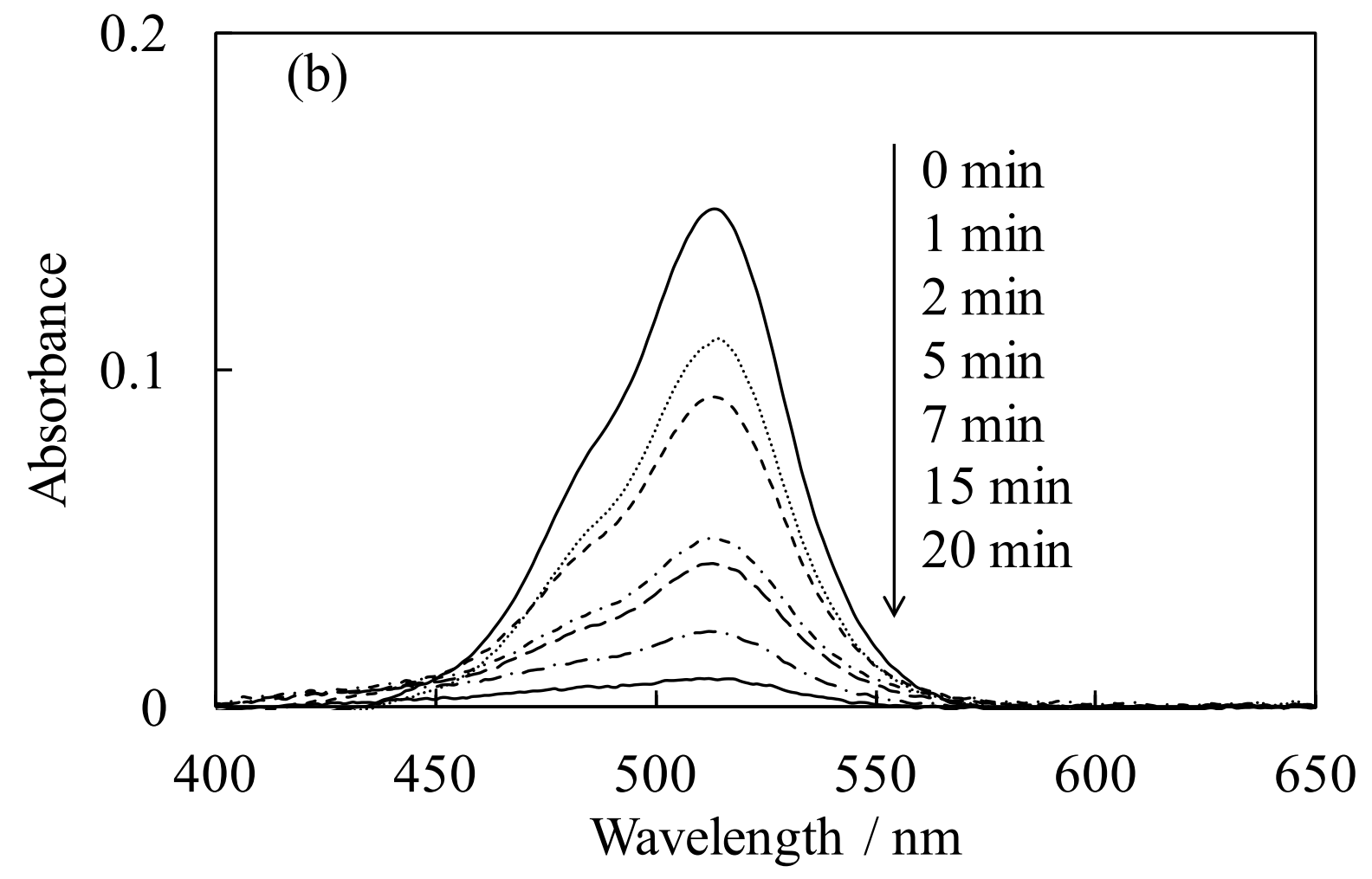


Figure 5

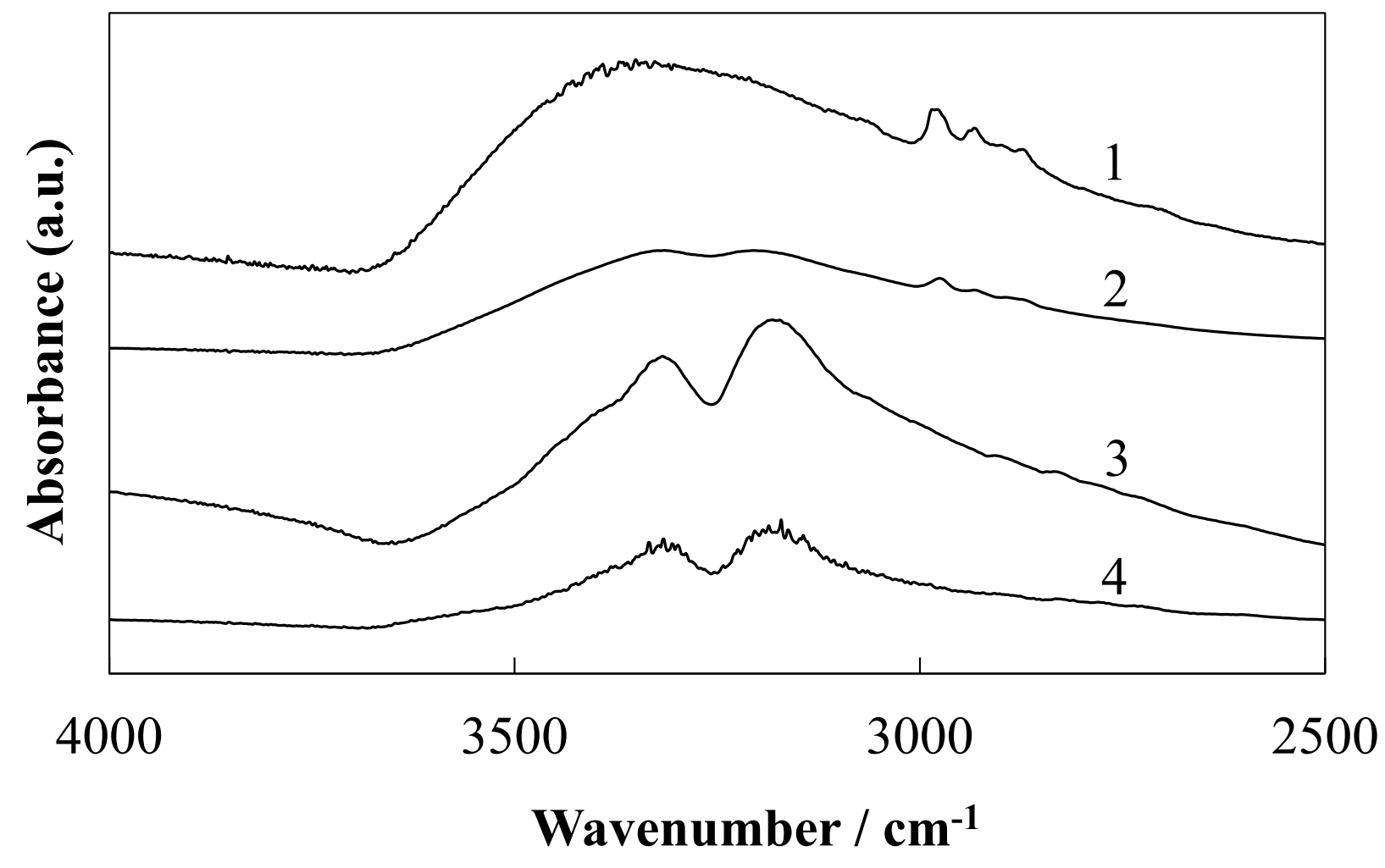


Figure 6

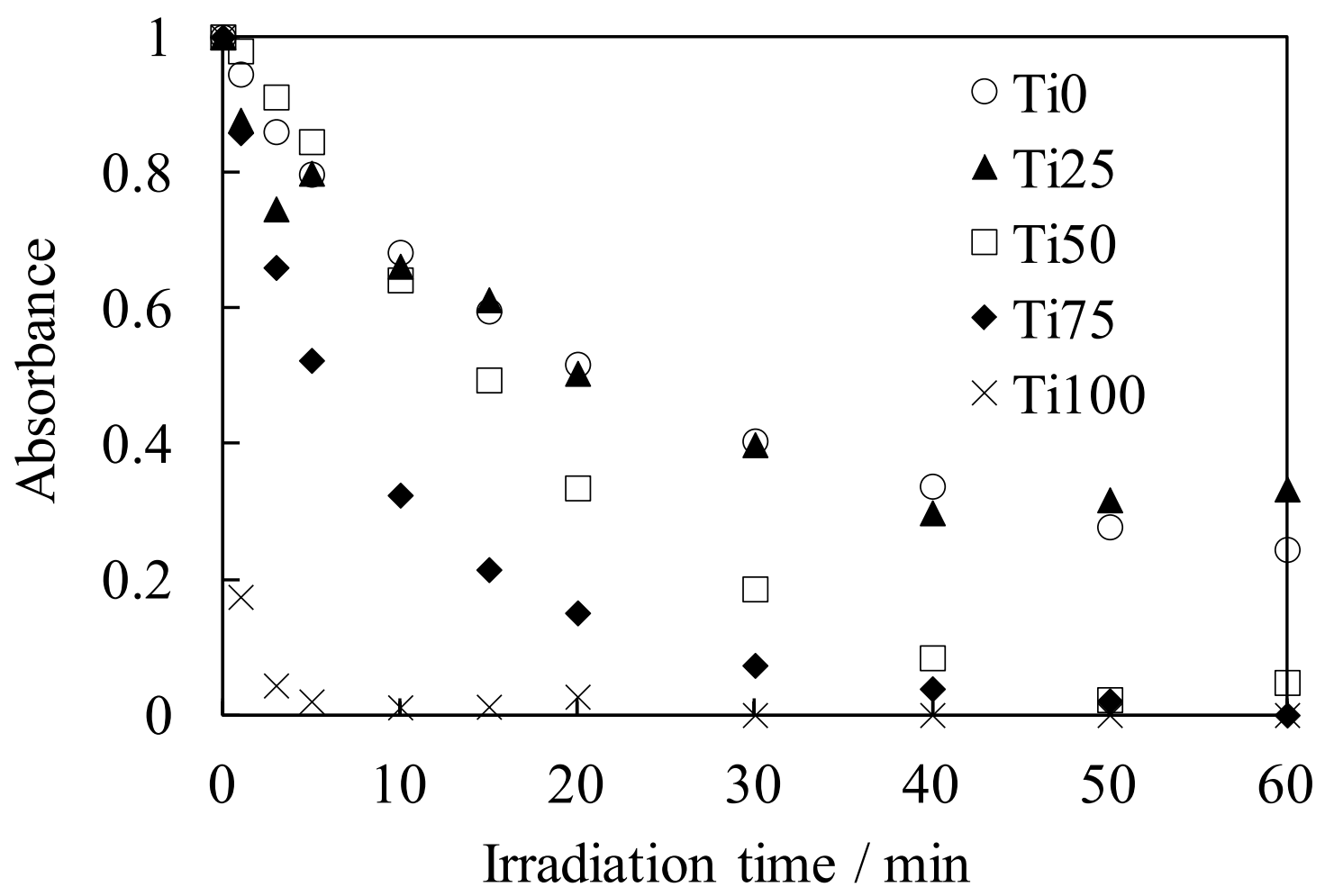


Figure 7
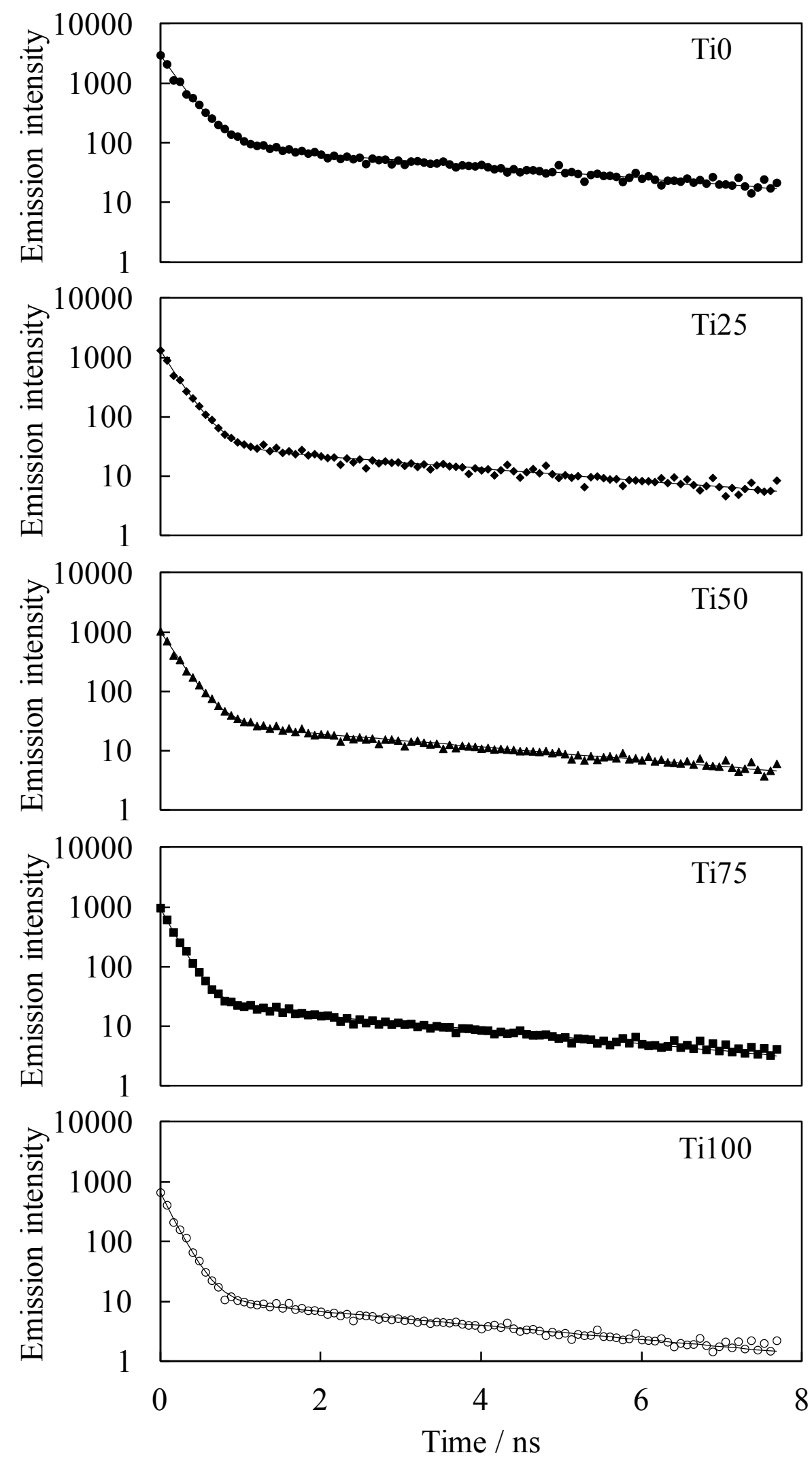
Figure 8

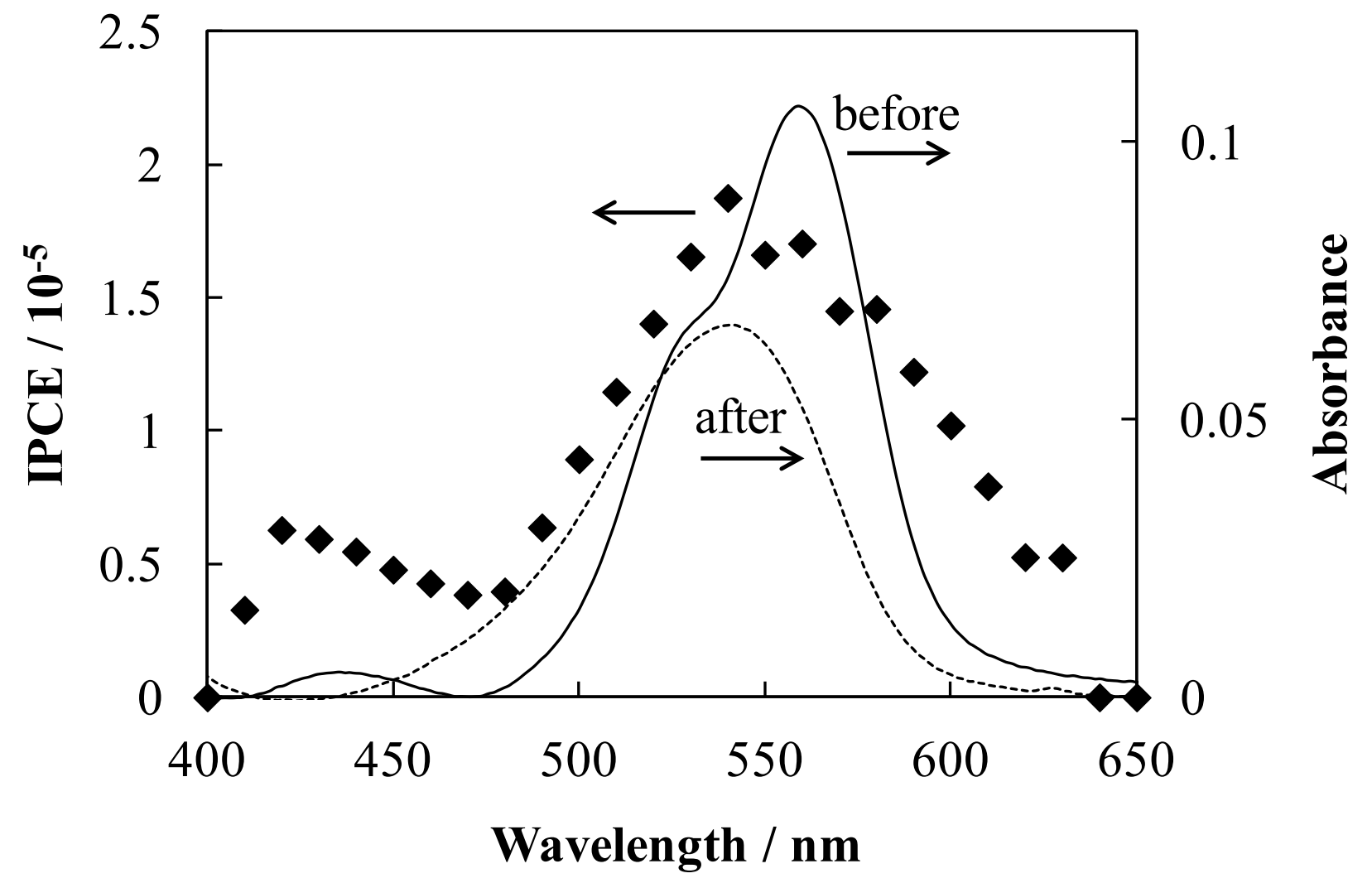


Figure 9

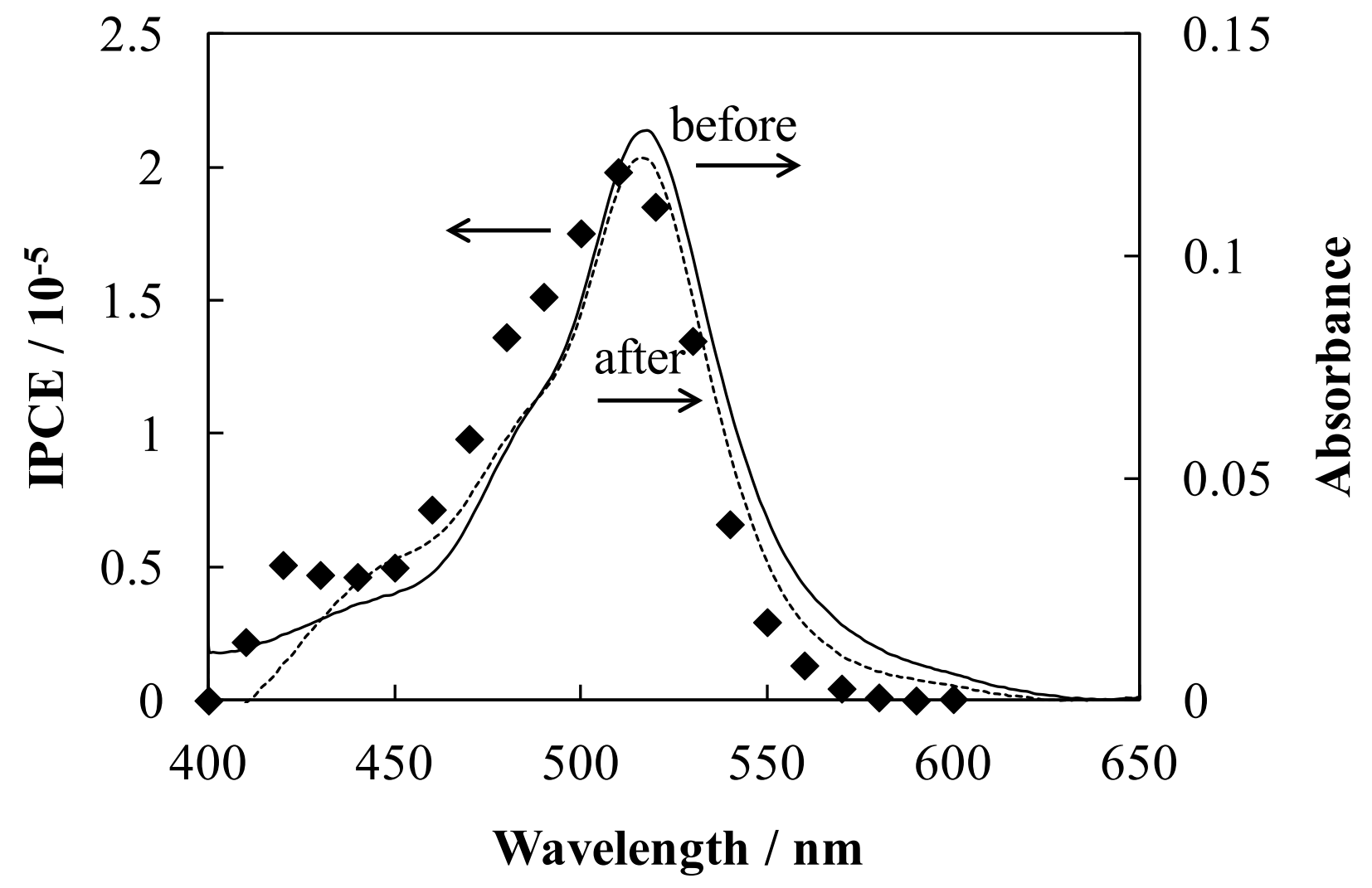


Figure 10

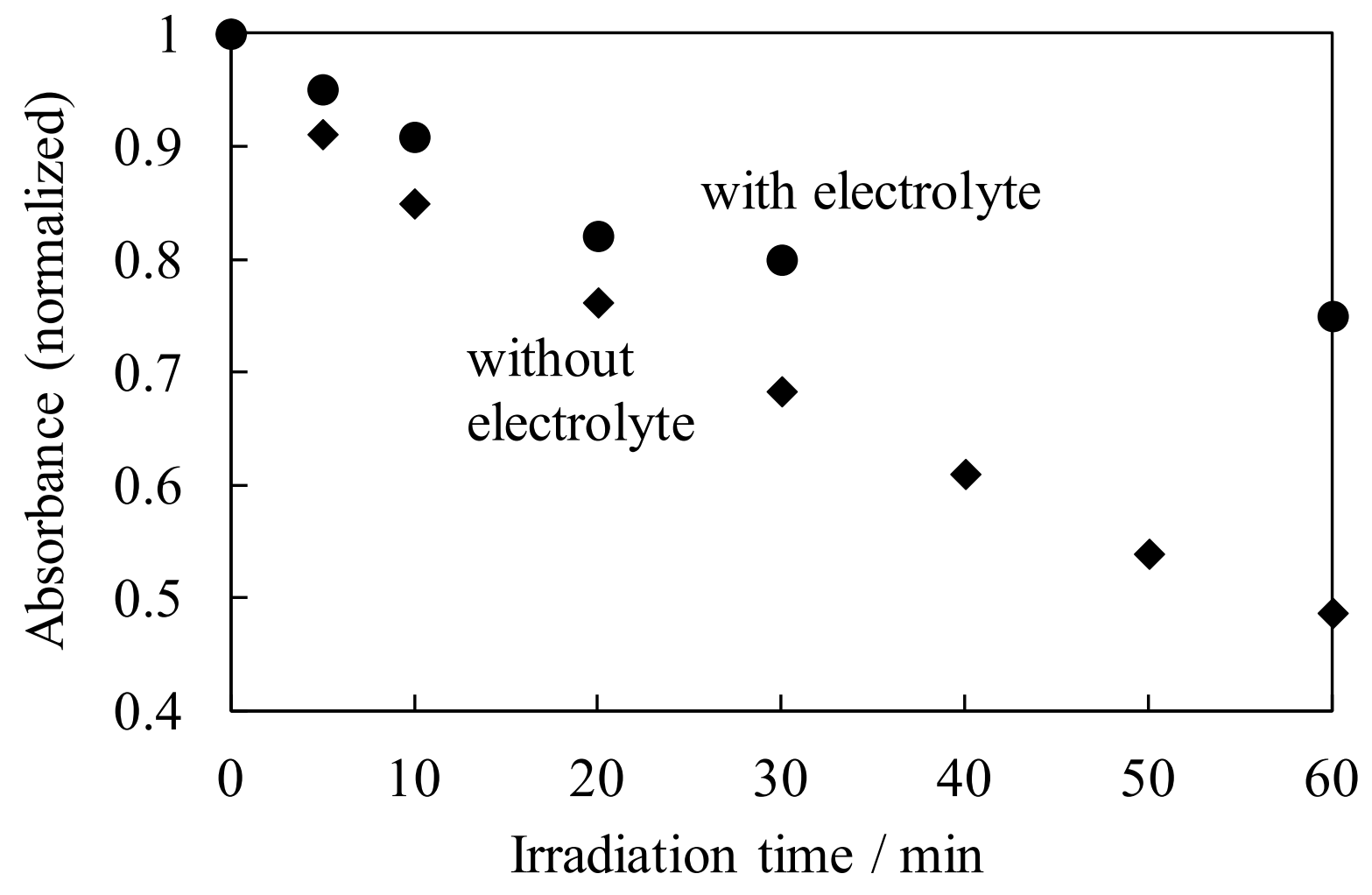

